\title{
Red mud catalyzed one-pot synthesis of nitriles from aldehydes and hydroxylamine hydrochloride under microwave irradiation
}

\author{
S. Hadi Khezri, ${ }^{\text {a Nahal Azimi, }}$, Mehrdad Mohammed-Vali, ${ }^{\text {a }}$ Bagher Eftekhari-Sis, \\ Mohammed M. Hashemi, ${ }^{\text {a, }}$ Mohammed H. Baniasadi, ${ }^{b}$ and Fatemeh Teimouri ${ }^{c}$ \\ ${ }^{a}$ Department of Chemistry, Sharif University of Technology, P. O. Box 11365-9516, Tehran, Iran \\ ${ }^{b}$ R\&D Department, N.K. Process Co., North Kargar Ave., Zomorrod Ally, No.22, Tehran, Iran \\ ${ }^{c}$ Department of Chemistry, Islamic Azad University - Saveh Branch, P. O. 39187-366, Saveh, \\ Iran \\ E-mail: mhashemi@sharif.edu
}

\begin{abstract}
Red mud ( $\mathrm{RM}$ ) which emerges as a by-product from the alumina producing process has been used as an efficient catalyst for one-pot direct synthesis of nitrile compounds from aldehydes and hydroxylamine hydrochloride under microwave irradiation.
\end{abstract}

Keywords: Nitrile, red mud, microwave irradiation, aldehyde, hydroxylamine hydrochloride

\section{Introduction}

Nitrile compounds are important intermediates for the synthesis of pharmaceuticals, pesticides and dyes. ${ }^{1}$ There are several methods for synthesis of nitrile compounds such as substitution reactions of alkyl and aryl halides with metal cyanides, ${ }^{2}$ oxidation of primary amines, ${ }^{3}$ reaction of aldehydes and ammonia using $\mathrm{H}_{2} \mathrm{SO}_{4}{ }^{4}$ and $\mathrm{I}_{2}$, reaction of carboxylic acids with tosylamide in the presence of $\mathrm{PCl}_{5}{ }^{6}$ or $\mathrm{KSCN}^{7}$ dehydration of primary ${ }^{8}$ and secondary ${ }^{9}$ amides, dehydration of aldoximes with dehydrating agents such as cetyltrimethylammonium dichromate., ${ }^{10}$ 2,4,6trichloro[1,3,5]triazine, ${ }^{11}$ chloral (hydrate), ${ }^{12}$ thionyl chloride, ${ }^{13} \mathrm{PCl}_{5}{ }^{14}$, orthoester, ${ }^{15}$ $\left(\mathrm{CF}_{3} \mathrm{CO}\right)_{2} \mathrm{O},{ }^{16}$ triethylamine $/ \mathrm{SO}_{2},{ }^{17} \mathrm{SeO}_{2},{ }^{18}$ chlorosulfonyl isocyanate, ${ }^{19} \mathrm{PPh}_{3} / \mathrm{CCl}_{4},{ }^{20}$, Methyl N(triethylammoniumsulphonyl)carbamate (Burgess reagent), ${ }^{21} \mathrm{NaHSO}_{4} / \mathrm{SiO}_{2},{ }^{22} \mathrm{MeSO}_{2} \mathrm{Cl}^{23}$ $\mathrm{AlCl}_{3} / \mathrm{KCl}^{24}{ }^{24} \mathrm{NaI} / \mathrm{MeCN},{ }^{25} \mathrm{PCl}_{5} /$ alumina, ${ }^{26}\left[\mathrm{RuCl}_{2} \text { (p-cymene) }\right]_{2} /$ molecular sieves, ${ }^{27}$ silica gel ${ }^{28}$ and zeolite. ${ }^{29}$ It has been reported that $\mathrm{N}$-aminopyridines condense with aldehydes to yield the corresponding Schiff's bases that are converted in the gas-phase into nitriles. ${ }^{30}$ Conversion of aldehydes into nitriles via reaction of the former with hydroxylamine sulfonic acid has also been reported. ${ }^{31}$ Recently, conversion of arylhydrazonals into hydrazononitriles via the reaction of hydrazonals with hydroxylamine hydrochloride and sodium acetate has also been reported. The 
latter reaction has also been conducted in the microwave oven. ${ }^{32}$ Another reported method for nitrile compound synthesis is the one-pot reaction of aldehydes and hydroxylamine using pyridine, ${ }^{33} \mathrm{SeO}_{2},{ }^{34} \mathrm{H}_{3} \mathrm{PO}_{4},{ }^{35} \mathrm{H}_{2} \mathrm{SO}_{3},{ }^{36}$ formic acid, ${ }^{37}$ dicyclohexyl carbodiimide(DCC)/ $\mathrm{CuCl}_{2},{ }^{38}$ $\mathrm{MgSO}_{4} /$ toluenesulfonic acid, $^{39} \mathrm{~N}$-tosylimines, ${ }^{40}$ oxalylchloride, ${ }^{41}$ acetyl chloride/charcoal, ${ }^{42}$ polymer-supported reagent, ${ }^{43} \mathrm{~N}$-methylpyrrolidone, ${ }^{44}$ phthalic anhydride/ $\mathrm{Et}_{3} \mathrm{~N}^{45}{ }^{45} \mathrm{NaN}_{3} / \mathrm{AlCl}_{3}{ }^{46}$ $\mathrm{ClSi}\left(\mathrm{N}_{3}\right)_{3},{ }^{47}\left(\mathrm{CH}_{3}\right)_{3} \mathrm{SiN}_{3},{ }^{48}$ anhydrous $\mathrm{K}_{2} \mathrm{~S}_{2} \mathrm{O}_{8} /$ alumina ${ }^{49}$ and acetic anhydride. ${ }^{50}$ Some of these methods suffer from limitations such as corrosive, toxic, expensive or commercially unavailable reagents, excess stoichiometric and no reusability of reagents, vigorous reaction conditions, unsatisfactory yields and tedious work up. ${ }^{4-50}$ Dewan, et al. recently reported a one-pot conversion of benzaldehyde and hydroxylamine to nitriles using $\mathrm{Na}_{2} \mathrm{SO}_{4},{ }^{51} \mathrm{NaHCO}_{3},{ }^{51}$ silica gel, ${ }^{52}$ Montmorillonite $\mathrm{K}-10,{ }^{53}$ or $\mathrm{KSF}^{53}$ in dry media under microwave irradiation.

Red mud (RM) emerges as a by-product from the caustic leaching of bauxite to produce alumina. Its major constituents are $\mathrm{Fe}_{2} \mathrm{O}_{3}, \mathrm{Al}_{2} \mathrm{O}_{3}, \mathrm{SiO}_{2}, \mathrm{Na}_{2} \mathrm{O}, \mathrm{TiO}_{2}, \mathrm{MgO}$ and $\mathrm{CaO}$. It contains small amounts of $\mathrm{Zr}, \mathrm{Y}$, Th and $\mathrm{U}$ elements and trace amounts of rare earth elements. ${ }^{54}$ The use of RM as an efficient catalyst for various transformations such as hydrogenation, ${ }^{55}$ dehydrochlorination of tetrachloroethylene ${ }^{56}$ and transformation of heavy hydrocarbons to fuel $\mathrm{oil}^{57}$ has been well documented in the literature.

Recently, we have reported that RM catalyzes the Knoevenagel and aldol condensation reactions. ${ }^{58}$ The results and importance of one-pot reactions ${ }^{59}$ encouraged us to study the one-pot synthesis of nitrile compounds from aldehydes and hydroxylamine hydrochloride using RM. As part of our research on chemical transformations, ${ }^{60}$ in this paper we report a simple and environmentally benign methodology for the one-pot direct synthesis of nitrile compounds from aldehydes and hydroxylamine hydrochloride using RM as a catalyst. The overall reaction is shown in Scheme 1.

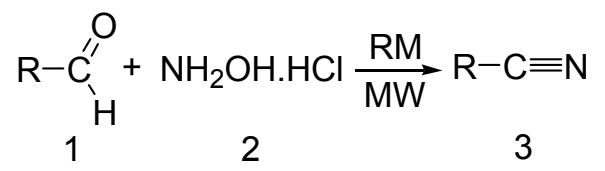

\section{Scheme 1}

\section{Results and Discussion}

The RM catalyzed one-pot direct synthesis of nitrile compounds was first studied using benzaldehyde and hydroxylamine hydrochloride under microwave irradiation; benzonitrile was isolated in high yield.

To examine the optimal conditions for this reaction using RM as the catalyst, we carried out experiments a-g (Table 1). According to experiments a-c (Table 1) at room temperature the aldoxime was the only product, and an increase in the temperature led to isolation of the nitrile, 
but in low yield. Experiments $\mathrm{c}$ and $\mathrm{g}$ (Table 1) show that microwave irradiation is essential for the best result. Experiments $d$ and $g$ (Table 1) described that the nitrile product was obtained in high yield and short reaction time when the $\mathrm{RM}$ was activated $\left(100{ }^{\circ} \mathrm{C}\right.$ at oven for $\left.8 \mathrm{~h}\right)$. Experiments e-f (Table 1) show the effect of microwave irradiation power; the optimum power of irradiation was determined to be $1000 \mathrm{~W}$. Experiment $\mathrm{g}$ (Table 1) describes the yields of three consecutive reactions of benzaldehyde and hydroxylamine hydrochloride leading to benzonitrile using RM as catalyst. In these experiments the product was isolated by filtration, the solid residue was washed with ethyl acetate and the remaining $\mathrm{RM}$ reloaded with fresh reagent for future runs after reactivation $\left(100^{\circ} \mathrm{C}\right.$ at oven for $\left.8 \mathrm{~h}\right)$. No considerable decrease in the yield was observed, demonstrating that RM can be reused as a catalyst in the one-pot synthesis of benzonitrile.

Table 1. Optimization of reaction conditions

\begin{tabular}{|c|c|c|c|}
\hline Entry & Reaction condition & Time $(\min )$ & Yield $(\%)^{\mathrm{a}}$ \\
\hline $\mathrm{a}$ & Inactivated RM, r.t. & 30 & $-\mathrm{b}^{\mathrm{b}}$ \\
\hline $\mathrm{b}$ & Activated $\mathrm{RM}$ at $100^{\circ} \mathrm{C}$ for $8 \mathrm{~h}$, r.t. & 30 & $-----{ }^{b}$ \\
\hline $\mathrm{c}$ & Activated $\mathrm{RM}$ at $100^{\circ} \mathrm{C}$ for $8 \mathrm{~h}, 80^{\circ} \mathrm{C}$ (at oven) & 30 & 20 \\
\hline d & Inactivated RM, MW (1000W) & 5 & 38 \\
\hline e & Activated $\mathrm{RM}$ at $100^{\circ} \mathrm{C}$ for $8 \mathrm{~h}, \mathrm{MW}(600 \mathrm{~W})$ & 10 & 63 \\
\hline $\mathrm{f}$ & Activated $\mathrm{RM}$ at $100^{\circ} \mathrm{C}$ for $8 \mathrm{~h}, \mathrm{MW}(800 \mathrm{~W})$ & 7 & 70 \\
\hline $\mathrm{g}$ & Activated $\mathrm{RM}$ at $100^{\circ} \mathrm{C}$ for $8 \mathrm{~h}, \mathrm{MW}(1000 \mathrm{~W})$ & 3 & $97,95,96^{\mathrm{c}}$ \\
\hline $\mathrm{h}$ & Activated $\mathrm{RM}$ at $100^{\circ} \mathrm{C}$ for $8 \mathrm{~h}, \mathrm{MW}(1200 \mathrm{~W})$ & 3 & 95 \\
\hline
\end{tabular}

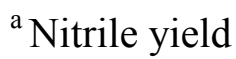

${ }^{\mathrm{b}}$ the isolated product is aldoxime

${ }^{\mathrm{c}}$ Catalyst was used over four runs

Reaction of hydroxylamine hydrochloride with different types of aromatic and aliphatic aldehydes using RM as a catalyst under MW irradiation was then investigated. The results are summarized in Table 2. The data in Table 2 clearly show that the reactions of different aldehydes give the corresponding nitriles in good to high yield with a short reaction time in dry media under MW irradiation.

\section{Conclusions}

In summary, it can be concluded that RM, that is the by-product of the Bayer process, can be used as an efficient, excellent and readily available reagent and a very inexpensive catalyst in nitrile synthesis from aldehydes and hydroxylamine hydrochloride under microwave irradiation. Furthermore, the reaction is a green process and the catalyst is recyclable for several uses. 
Table 2. One-pot direct synthesis of nitrile compounds from aldehydes and hydroxylamine hydrochloride using RM as a catalyst (Scheme 1).

\begin{tabular}{llll}
\hline Product & $\mathrm{R}$ & Time $($ min) & Yield (\%) \\
\hline $3 \mathrm{a}$ & $\mathrm{Ph}$ & 3 & 89 \\
$3 \mathrm{~b}$ & $4-\mathrm{ClC}_{6} \mathrm{H}_{4}$ & 4 & 91 \\
$3 \mathrm{c}$ & $2-\mathrm{ClC}_{6} \mathrm{H}_{4}$ & 5 & 76 \\
$3 \mathrm{~d}$ & 4- $\mathrm{FC}_{6} \mathrm{H}_{4}$ & 4 & 83 \\
$3 \mathrm{e}$ & 4- $\mathrm{MeC}_{6} \mathrm{H}_{4}$ & 3 & 90 \\
$3 \mathrm{f}$ & 3- $\mathrm{NO}_{2} \mathrm{C}_{6} \mathrm{H}_{4}$ & 5 & 78 \\
$3 \mathrm{~g}$ & 4- $\mathrm{NO}_{2} \mathrm{C}_{6} \mathrm{H}_{4}$ & 4 & 97 \\
$3 \mathrm{~h}$ & 4- $\mathrm{MeOC}_{6} \mathrm{H}_{4}$ & 2 & 91 \\
$3 \mathrm{j}$ & 4-(Me) ${ }_{2} \mathrm{NC}_{6} \mathrm{H}_{4}$ & 2 & 93 \\
$3 \mathrm{k}$ & 2-furyl & 3 & 82 \\
31 & 2-thienyl & 4 & 93 \\
$3 \mathrm{~m}$ & 3-pyridyl & 3 & 98 \\
$3 \mathrm{n}$ & 4-pyridyl & 3 & 89 \\
$3 \mathrm{o}$ & n-butyl & 5 & 71 \\
$3 \mathrm{p}$ & n-hexyl & 5 & 75 \\
$3 \mathrm{q}$ & n-octyl & 5 & 63 \\
\hline
\end{tabular}

\section{Experimental Section}

General Procedures. Melting points were measured on an Electrothermal 9100 apparatus and are uncorrected. IR spectra were recorded on a Shimadzu IR-470 spectrometer. ${ }^{1} \mathrm{H}$ NMR spectra were recorded on a BRUKER DRX-500 AVANCE spectrometer at 500.13. NMR spectra were obtained on solutions in $\mathrm{CDCl}_{3}$. The mixture of the reaction was irradiated with microwave (MW) laboratory reactor (ETHOS. MR) producing controlled irradiation. The chemicals used in this work were purchased from Fluka (Buchs, Switzerland) and Merck chemical companies. The RM that was used in this work was obtained from Indian gibbsite bauxite and chemical analysis of it was determined with XRF (BRUKER S4 EXPLORER). Table 3 shows these results.

Table 3. XRF analysis of RM used

\begin{tabular}{llllllll}
\hline Species & $\mathrm{Fe}_{2} \mathrm{O}_{3}$ & $\mathrm{Al}_{2} \mathrm{O}_{3}$ & $\mathrm{SiO}_{2}$ & $\mathrm{Na}_{2} \mathrm{O}$ & $\mathrm{TiO}_{2}$ & $\mathrm{CaO}$ & $\mathrm{ZrO}_{2}$ \\
\hline Content (W\%) & 25.13 & 19.51 & 18.5 & 11.04 & 3.79 & 3.06 & 0.45 \\
Species & $\mathrm{K} 2 \mathrm{O}$ & $\mathrm{MgO}$ & $\mathrm{MnO}$ & $\mathrm{SO}_{3}$ & $\mathrm{Cl}$ & $\mathrm{P}_{2} \mathrm{O}_{5}$ & $\mathrm{LOI}^{\mathrm{a}}$ \\
Content (W\%) & 0.39 & 0.22 & 0.07 & 1.23 & 0.33 & 0.27 & 16.01 \\
\hline
\end{tabular}

${ }^{\mathrm{a}}$ Loss on ignition 


\section{General procedure for nitrile preparation}

A mixture of an aldehyde $(1 \mathrm{mmol})$ and hydroxylamine hydrochloride $(1 \mathrm{mmol})$ and activated RM (3 g) were mixed thoroughly in a mortar and the mixture was transferred to a beaker and irradiated at $2450 \mathrm{MHz}\left(100 \%\right.$ power, $\left.1000 \mathrm{~W}, 80^{\circ} \mathrm{C}\right)$. The progress of the reaction was monitored by TLC using ethyl acetate: n-hexane $(20: 80, v / v)$ as solvent. The mixture was extracted with ethyl acetate and then filtered. The solvent was removed under reduced pressure to afford nitrile.

\section{Activation and recovery of $\mathrm{RM}$}

$\mathrm{RM}$ was activated at $100{ }^{\circ} \mathrm{C}$ for $8 \mathrm{~h}$ and cooled to room temperature in a desiccator under vacuum. For recovery of the RM, after washing twice with ethyl acetate, it was activated again at $100{ }^{\circ} \mathrm{C}$ before reuse.

\section{Selected data for compounds 3a-3q}

Benzonitrile (3a). Bp 187-190 ${ }^{\circ} \mathrm{C}\left[\text { lit. } 190{ }^{\circ} \mathrm{C}\right]^{61}$; IR (KBr) $\left(v_{\max }, \mathrm{cm}^{-1}\right): 3031,2232 ;{ }^{1} \mathrm{H}$ NMR $\left(\mathrm{CDCl}_{3}, 500 \mathrm{MHz}\right): \delta_{\mathrm{H}} 7.55-7.61(5 \mathrm{H}, \mathrm{m}, \mathrm{H}-\mathrm{Ar})$.

p-Chlorobenzonitrile (3b). Mp 91-93 ${ }^{\circ} \mathrm{C}\left[\text { lit. } 95-96{ }^{\circ} \mathrm{C}\right]^{62}$; IR (KBr) $\left(v_{\max }, \mathrm{cm}^{-1}\right): 3069,2228 ;{ }^{1} \mathrm{H}$ NMR $\left(\mathrm{CDCl}_{3}, 500 \mathrm{MHz}\right): \delta_{\mathrm{H}} 7.37(2 \mathrm{H}, \mathrm{d}, J=8.4 \mathrm{~Hz}, \mathrm{H}-\mathrm{Ar}), 7.47$ (2H, d, $\left.J=8.4 \mathrm{~Hz}, \mathrm{H}-\mathrm{Ar}\right)$.

m-Chlorobenzonitrile (3c). Mp 43-46 ${ }^{\circ} \mathrm{C}\left[\text { lit. } 46-47{ }^{\circ} \mathrm{C}\right]^{63}$; IR (KBr) $\left(v_{\max }, \mathrm{cm}^{-1}\right): 3032,2231 ;{ }^{1} \mathrm{H}$ NMR $\left(\mathrm{CDCl}_{3}, 500 \mathrm{MHz}\right): \delta_{\mathrm{H}} 7.50-7.64(5 \mathrm{H}, \mathrm{m}, \mathrm{H}-\mathrm{Ar})$.

p-Fluorobenzonitrile (3d). $\mathrm{Mp} 33-36{ }^{\circ} \mathrm{C}\left[\right.$ lit. $\left.35-36{ }^{\circ} \mathrm{C}\right]{ }^{64}$; IR (KBr) $\left(v_{\max }, \mathrm{cm}^{-1}\right): 3086,2238 ;{ }^{1} \mathrm{H}$ NMR $\left(\mathrm{CDCl}_{3}, 500 \mathrm{MHz}\right): \delta_{\mathrm{H}} 7.68(2 \mathrm{H}, \mathrm{m}, \mathrm{H}-\mathrm{Ar}), 7.19$ (2H, m, H-Ar).

p-Tolunitrile (3e). Bp 215-217 ${ }^{\circ} \mathrm{C}\left[\text { lit. } 218{ }^{\circ} \mathrm{C}\right]^{61}$; IR (KBr) $\left(v_{\max }, \mathrm{cm}^{-1}\right): 3039,2229$; ${ }^{1} \mathrm{H}$ NMR $\left(\mathrm{CDCl}_{3}, 500 \mathrm{MHz}\right): \delta_{\mathrm{H}} 7.23(2 \mathrm{H}, \mathrm{d}, J=7.4 \mathrm{~Hz}, \mathrm{H}-\mathrm{Ar}), 7.47(2 \mathrm{H}, \mathrm{d}, J=7.4 \mathrm{~Hz}, \mathrm{H}-\mathrm{Ar})$.

m-Nitrobenzonitrile (3f). Mp $115-117{ }^{\circ} \mathrm{C}\left[\right.$ lit. $\left.115^{\circ} \mathrm{C}\right]{ }^{65}$; IR (KBr) $\left(v_{\max }, \mathrm{cm}^{-1}\right): 3036,2238 ;{ }^{1} \mathrm{H}$ NMR $\left(\mathrm{CDCl}_{3}, 500 \mathrm{MHz}\right): \delta_{\mathrm{H}} 7.70(1 \mathrm{H}, \mathrm{m}, \mathrm{H}-\mathrm{Ar}), 7.90(1 \mathrm{H}, \mathrm{d}, J=9.0 \mathrm{~Hz}, \mathrm{H}-\mathrm{Ar}), 8.46$ (2H, m, HAr).

p-Nitrobenzonitrile (3g). Mp 146-149 ${ }^{\circ} \mathrm{C}\left[\right.$ lit. $\left.147{ }^{\circ} \mathrm{C}\right]{ }^{61}$; IR $(\mathrm{KBr})\left(\mathrm{v}_{\max }, \mathrm{cm}^{-1}\right): 3076,2234 ;{ }^{1} \mathrm{H}$ $\operatorname{NMR}\left(\mathrm{CDCl}_{3}, 500 \mathrm{MHz}\right): \delta_{\mathrm{H}} 7.92(2 \mathrm{H}, \mathrm{d}, J=8.0 \mathrm{~Hz}, \mathrm{H}-\mathrm{Ar}), 8.37$ (2H, d, J=8.0 Hz, H-Ar).

p-Methoxybenzonitrile (3h). Mp 57-59 ${ }^{\circ} \mathrm{C}$ [lit. 62-63 $\left.{ }^{\circ} \mathrm{C}\right]^{66}$; IR (KBr) $\left(v_{\max }, \mathrm{cm}^{-1}\right)$ : 3068, 2219; ${ }^{1} \mathrm{H}$ NMR $\left(\mathrm{CDCl}_{3}, 500 \mathrm{MHz}\right): \delta_{\mathrm{H}} 3.73(3 \mathrm{H}, \mathrm{s}, \mathrm{Me}), 6.95(2 \mathrm{H}, \mathrm{d}, J=9.1 \mathrm{~Hz}, \mathrm{H}-\mathrm{Ar}), 7.40$ (2H, d, $J=$ 9.1 Hz, H-Ar).

p-Dimethylaminobenzonitrile (3j). $\mathrm{Mp} 69-71{ }^{\circ} \mathrm{C}\left[1 \mathrm{it} .75-77{ }^{\circ} \mathrm{C}\right]^{67}$; IR (KBr) $\left(v_{\max }, \mathrm{cm}^{-1}\right): 2909$, 2261; ${ }^{1} \mathrm{H}$ NMR $\left(\mathrm{CDCl}_{3}, 500 \mathrm{MHz}\right): \delta_{\mathrm{H}} 3.02$ (6H, s, Me), 6.63 (2H, d, J=8.2 Hz, H-Ar), 7.43 $(2 \mathrm{H}, \mathrm{d}, J=8.2 \mathrm{~Hz}, \mathrm{H}-\mathrm{Ar})$.

2-Furonitrile (3k). Mp 146-148 ${ }^{\circ} \mathrm{C}$ [lit. 146-147 $\left.{ }^{\circ} \mathrm{C}\right]{ }^{68}$; IR (KBr) $\left(v_{\max }, \mathrm{cm}^{-1}\right): 2981,2236 ;{ }^{1} \mathrm{H}$ NMR $\left(\mathrm{CDCl}_{3}, 500 \mathrm{MHz}\right): \delta_{\mathrm{H}} 6.36(1 \mathrm{H}, \mathrm{m}, \mathrm{H}$-furyl $), 6.86(1 \mathrm{H}, \mathrm{d}, J=3.6 \mathrm{~Hz}, \mathrm{H}$-furyl $), 7.38(1 \mathrm{H}$, $\mathrm{d}, J=1.8 \mathrm{~Hz}, \mathrm{H}$-furyl). 
2-Thiophenecarbonitrile (3I). Mp 125-134 ${ }^{\circ} \mathrm{C}$ (decomp) [lit. $\left.192{ }^{\circ} \mathrm{C}\right]^{69}$; IR (KBr) $\left(v_{\max }, \mathrm{cm}^{-1}\right)$ : 3067, 2238; ${ }^{1} \mathrm{H} \mathrm{NMR}\left(\mathrm{CDCl}_{3}, 500 \mathrm{MHz}\right): \delta_{\mathrm{H}} 6.96(1 \mathrm{H}, \mathrm{m}, \mathrm{H}$-thienyl), $7.42(1 \mathrm{H}, \mathrm{m}, \mathrm{H}$-thienyl), 7.49 (1H, m, H-thienyl).

Nicotinonitrile (3m). Mp $50{ }^{\circ} \mathrm{C}\left[\text { lit. } 50{ }^{\circ} \mathrm{C}\right]^{70}$; IR (KBr) $\left(v_{\max }, \mathrm{cm}^{-1}\right): 3036,2232 ;{ }^{1} \mathrm{H}$ NMR $\left(\mathrm{CDCl}_{3}, 500 \mathrm{MHz}\right): \delta_{\mathrm{H}} 7.48(1 \mathrm{H}, \mathrm{m}, \mathrm{H}-$ pyridil $), 8.00(1 \mathrm{H}, \mathrm{d}, J=1.9 \mathrm{~Hz}, \mathrm{H}-$ pyridil $), 8.85(1 \mathrm{H}, \mathrm{d}$, $J=1.9 \mathrm{~Hz}, \mathrm{H}$-pyridil), 8.91 (1H, m, H-pyridil).

iso-Nicotinonitrile (3n). $\mathrm{Mp} 76{ }^{\circ} \mathrm{C}\left[\text { lit. } 75-77^{\circ} \mathrm{C}\right]^{71}$; IR (KBr) $\left(v_{\max }, \mathrm{cm}^{-1}\right): 3084,2244 ;{ }^{1} \mathrm{H}$ NMR $\left(\mathrm{CDCl}_{3}, 500 \mathrm{MHz}\right): \delta_{\mathrm{H}} 7.55(2 \mathrm{H}, \mathrm{d}, J=4.1 \mathrm{~Hz}, \mathrm{H}$-pyridil), $8.83(2 \mathrm{H}, \mathrm{d}, J=4.1 \mathrm{~Hz}, \mathrm{H}$-pyridil).

Pentanenitrile (3o). Bp 139-141 ${ }^{\circ} \mathrm{C}\left[\right.$ lit. $\left.\left.142{ }^{\circ} \mathrm{C}\right]\right]^{72}$; IR (KBr) $\left(v_{\max }, \mathrm{cm}^{-1}\right): 2742,2683,2247 ;{ }^{1} \mathrm{H}$ NMR ( $\left.\mathrm{CDCl}_{3}, 500 \mathrm{MHz}\right): \delta_{\mathrm{H}} 0.96(3 \mathrm{H}, \mathrm{t}, J=2.8 \mathrm{~Hz}, \mathrm{H}-\mathrm{Aliph}), 1.68$ (2H, m, H-Aliph), $1.50(2 \mathrm{H}$, m, H-Aliph), 2.34 (2H, t, $J=2.8 \mathrm{~Hz}$, H-Aliph).

Heptanenitrile (3p). Bp $243{ }^{\circ} \mathrm{C}$ (decomp) [lit. $\left.253{ }^{\circ} \mathrm{C}\right]^{3 \mathrm{a}}$; IR (KBr) $\left(v_{\max }, \mathrm{cm}^{-1}\right): 2968,2933$, 2247; ${ }^{1} \mathrm{H}$ NMR $\left(\mathrm{CDCl}_{3}, 500 \mathrm{MHz}\right): \delta_{\mathrm{H}} 0.90(3 \mathrm{H}, \mathrm{t}, J=2.8 \mathrm{~Hz}, \mathrm{H}-\mathrm{Aliph}), 1.32$ (4H, m, H-Aliph), 1.46 (2H, m, H-Aliph), 1.65 (2H, m, H-Aliph), 2.34 (2H, t, $J=2.8 \mathrm{~Hz}$, H-Aliph).

Undecanenitrile (3q). $\mathrm{Bp} 236{ }^{\circ} \mathrm{C}$ (decomp) [lit. $\left.268{ }^{\circ} \mathrm{C}\right]^{73}$; IR (KBr) $\left(v_{\max }, \mathrm{cm}^{-1}\right): 2966,2927$, 2247; ${ }^{1} \mathrm{H}$ NMR $\left(\mathrm{CDCl}_{3}, 500 \mathrm{MHz}\right): \delta_{\mathrm{H}} 0.89(3 \mathrm{H}, \mathrm{t}, J=2.5 \mathrm{~Hz}, \mathrm{H}-\mathrm{Aliph}), 1.27$ (14H, m, H-Aliph), $1.65(2 \mathrm{H}, \mathrm{m}, \mathrm{H}-\mathrm{Aliph}), 2.32$ (2H, t, $J=2.9 \mathrm{~Hz}, \mathrm{H}-$ Aliph).

\section{Acknowledgements}

We gratefully acknowledge financial support from the Sharif University of Technology.

\section{References}

1. (a) Friedrich, K.; Wallenfels, K. In The Chemistry of Cyano Group; Rappoport, Z. Ed.; Interscience: New York, 1970; p 92. (b) Fabiani, M. E. Drug News Perspect 1999, 12, 207. (c) Iqbal, A.; Cassar, L. Eur. Pat. Appl. 061,426; 1983: Chem. Abstr. 1983, 98, 73838n. (d) Wittenberger, S. J.; Donner, B. G. J. Org. Chem. 1993, 58, 4139. (e) Chihiro, M.; Nagamoto, H.; Tekemura, I.; Kitano, K.; Komatsu, H.; Sekiguchi, K.; Tabusa, F.; Mori, T.; Tominaga, M.; Yabuuchi, Y. J. Med. Chem. 1995, 38, 353.

2. (a) Mowry, D. T. Chem. Rev. 1948, 42, 189. (b) Kondo, Y.; Sugiyama, Y.; Tanaka, N. U.S. Patent 6,331, 628 B1, 2001. (c) Tagagi, K. Bull. Inst. Chem. Res. 1989, 67, 136.

3. (a) Semmelhack, M. F.; Schmid, C. R. J. Am. Chem. Soc. 1983, 105, 6732. (b) Chen, F. E.; Kuang, Y. Y.; Dai, H. F.; Lu, L.; Huo, M. Synthesis 2003, 2629. (c) Yamaguchi, K.; Mizuno, N. Angew. Chem. 2003, 115, 1518. (d) Nicolaou, K. C.; Mathison, C. J. N. Angew. Chem. 2005, 117, 6146.

4. Blatter, H. M.; Lukaszbewski, H.; De Stevens, G. J. Am. Chem. Soc.1961, 83, 2203. 
5. (a) Huimou, L.; Yiqun, L. CJI 2005, 7, 4. (b) Talukdar, S.; Hsu, J. L.; Chou, T. C.; Fang, J. M. Tetrahedron Lett. 2001, 42, 1103.

6. Miller, C. S. Org Synth. 1955, Coll. Vol III, 646.

7. Letts, E. A.; Ber. 1872, 5, 669.

8. (a) Baxendale, I. R.; Ley, S. V.; Sneddon, F. H. Synlett 2002, 5, 775. (b) Kuo, C. W.; Zhu, J. L.; Wu, J. D.; Chu, C. M.; Yao, C. F.; Shia, K. S. Chem. Commun. 2007, 301.

9. (a) Pechmann, H. Ber. 1900, 33, 611. (b) Phillips, B. A.; Fodor, G.; Gal, J.; Letourneau, F.; Ryan, J. J. Tetrahedron 1973, 29, 3309.

10. Sahu, S.; Patel, S.; Mishra, B. K. Synth. Commun. 2005, 35, 3123.

11. Luca, L. D.; Giacomelli, G.; Porcheddu, A. J. Org. Chem. 2002, 67, 6272.

12. Chandrasekhar, S.; Gopalaiah, K. Tetrahedron Lett. 2003, 44, 755.

13. (a) Olah, G. A.; Narang, S. C.; Garcia-Luma A. Synthesis 1980, 659. (b) Krynitsky, J. A.; Carhart, H. M. Org Synth. 1963, Coll. Vol IV, 436.

14. Koza, S. J. K. New Experimental Chemistry Course; Maruzen Co., Ltd., 1977; Vol. 14III, pp 1466.

15. Rogik, M. M.; Van Peppen, J. F.; Klein, K. P.; Demmin, T. R. J. Org. Chem. 1974, 39, 23.

16. Hendrickson, J. B.; Bair, K. W.; Keehn, P. M. Tetrahedron Lett. 1976, 17, 603.

17. Olah, G. A.; Vankar, Y. D. Synthesis 1978, 702.

18. Sosnovsky, G.; Krogh, J. A. Synthesis 1978, 703.

19. Olah, G. A.; Vankar, Y. D.; Garcia-Luna, A. Synthesis 1979, 227.

20. Kim, J. N.; Chung, K. H.; Pyn, E. K. Synth. Commun. 1990, 20, 2785.

21. Binoy, J.; Sulatha, M. S.; Madhavan Pillai, P.; Prathapan, S. Synth. Commun. 2000, 30, 1509.

22. Das, B.; Madhusudhan, P.; Venkataiah, B. Synlett 1999, 1569.

23. (a) Sharghi, H.; Sarvari, M. H. Tetrahedron 2002, 58, 10323. (b) Sharghi, H.; Sarvari, M. H. Synthesis 2003, 243.

24. Boruah, M.; Konwar, D. J. Org. Chem. 2002, 67, 7138.

25. Ballini, R.; Fiorini, D.; Palmieri, A. Synlett 2003, 1841.

26. Niknam, K.; Karami, B.; Kiasat, A. R. Bull. Korean Chem. Soc. 2005, 26, 975.

27. Yang, A. H.; Chang, S. Org. Lett. 2001, 3, 4209.

28. Dewan, S. K.; Singh, R. Synth. Commun. 2003, 33, 385.

29. (a) Hegedüs, A.; Cwik, A.; Hell, Z.; Horváth, Z.; Esek, Á.; Uzsokic, M. Green Chem. 2002, 4, 618. (b) Rao, M. N.; Kumar, P.; Garyali, K. Org. Prep. Proceed. Int. 1989, 21, 230. (c) Srinivas, K. V. N. S.; Reddy, B. E.; Das, B. Synlett 2002, 625.

30. (a) Al-Awadi, N. A.; Elnagdi, M. H.; Mathew, T.; El-Gamry, I.; Abdel Khalik, M. Int. J. of Chem. Kinet. 1996, 28, 741. (b) Al-Awadi, N. A.; Elnagdi, M. H.; Mathew, T.; Abdel Khalik, M. Int. J. of Chem. Kinet. 1996, 28, 749.

31. Al-Awadi, N. A.; Elnagdi, M. H.; Kaul, K.; Illingoran, S.; El-Dusouqui, O. Tetrahedron 1998, 54, 4633.

32. El-Dusouqui, O. M. E.; Abdelkhalik, M. M.; Al-Awadi, N. A.; Dib, H. H.; George, B. J.; Elnagdi, M. H. J. Chem. Res. 2006, 5, 295. 
33. Saednya, A. Synthesis 1982, 190.

34. Sosnovsky, G.; Krogh, J. A.; Umhoefer, S. G. Synthesis 1979, 722.

35. Ganboa, I.; Palomo, C. Synth. Commun. 1983, 13, 999.

36. (a) Streith, J.; Fizet, C.; Fritz, H. Helv. Chim. Acta. 1976, 59, 2786. (b) Fizet, C.; Streith, J. Tetrahedron Lett., 1974, 15, 3187.

37. (a) Olah, G. A.; Keumi, T. Synthesis 1979, 1120. (b) Feng, J. C.; Lin, B.; Dai, L.; Bian, N. S. Syn. Commun. 1998, 28, 3765. (c) BASF AG Ger. Offen. Appl. 1970, 2014984, 19700328.

38. Vowinkel, E.; Bartel, J. Chem. Ber. 1974, 107, 1221.

39. Ganboa, I.; Palomo, C. Synth. Commun. 1983, 13, 219.

40. Glass, R. S.; Hoy, R. C. Tetrahedron Lett. 1976, 17, 1781.

41. Movassagh, B.; Fazeli, A. Synth. Commun 2007, 37, 623.

42. Sharghi H.; Hosseini Saravi,M. J. Ir. Chem. Soc. 2004, 1, 28.

43. Baxendale, I. R.; Ley, S. V.; Sneddon, H. F. Synlett 2002, 775.

44. Kumar, H. M. S.; Reddy, B. V. S.; Reddy, P. T.; Yadav, J. S. Synthesis 1999, 586.

45. Wang, E. C.; Lin, G. J. Tetrahedron Lett. 1998, 39, 4047.

46. Suzuki, H.; Nakaya, C. Synthesis 1992, 641.

47. Elmorsy, S. S.; El-Ahl, A. S.; Soliman, H.; Amer, F. A. Tetrahedron Lett. 1995, 15, 2639.

48. Nishiyama, K.; Oba, M.; Watanabe, A. Tetrahedron 1987, 43, 693.

49. Bose, D. S.; Narsaiah, A. V. Tetrahedron Lett. 1998, 39, 6533.

50. (a) Lee, J. C.; Yoon, J. M.; Baek, J. W. Bull. Korean Chem. Soc. 2007, 28, 29. (b) Fahlhage, D. W. U.S. Patent 4,235, 807, 1980.

51. Dewan, S. K.; Singh, R.; Kumar, A. Arkivoc 2006, (ii), 41.

52. Dewan, S. K.; Singh, R. Synth. Commun. 2003, 33, 385.

53. (a) Dewan, S. K.; Singh, R.; Kumar, A. Synth. Commun 2004, 2025.

54. (a) Pera, J.; Boumaza, R.; Ambroise, J. Cement and Concrete Research 1997, 27, 1513. (b) Yalcin, N.; Sevinc, V. Ceramics International 2000, 26, 485.

55. (a) Alvarez, J.; Rosal, R.; Sastre, H.; Diez, F. V. Appl. Catal. A Gen., 1998, 167, 215. (b) Llano, J. J.; Rosal, R.; Sastre, H.; Diez F. V. Fuel 1994, 73, 688.

56. Ordonez, S.; Sastre, H.; Diez, F. V. J. Hazard. Mater. 2001, B81, 103.

57. Cakici, A. I.; Yanik, J.; UÇar, S.; Karayildirim, T.; Anil, H. J. Material Cycles and Waste Management 2004, 6, 20.

58. Khezri, S. H.; Mohmmed-vali,; M. Eftekhari-sis, B.; Hashemi, M. M. Green Chem. Lett. Rev. Accepted for publication.

59. (a) Rao, D. V. N. S.; Dandala, R.; Lenin, R.; Sivakumaran, M.; Shivashankar, S.; Naidu, A. Arkivoc 2007, (xiv), 34. (b) Azizian, J.; Mohammadizadeh, M. R.; Zomorodbakhsh, S.; Mohammadi, A. A.; Karimi, A. R. Arkivoc 2007, (xv), 24. (c) Locher, C.; Peerzada, N. Arkivoc 2000, (1), 14. (d) Kaboudin, B.; Karimi, M. Arkivoc 2007, (xiii), 124. (e) Mahmoodi, N. O.; Khodaee, Z. Arkivoc 2007, (iii), 29. (f) Stanetty, P.; Kasemann, O.; Mereiter, K.; Mihovilovic, M. D. Arkivoc 2005, (v), 83. (g) Filice, M.; Ubiali, D.; Pagani, G.; Terreni, M.; Pregnolato, M. Arkivoc 2006, (viii), 66. 
60. (a) Hashemi, M. M.; Eftekhari-Sis, B.; Abdollahifar, A.; Khalili. B. Tetrahedron, 2006, 62, 672. (b) Eftekhari-Sis, B.; Abdollahifar, A.; Hashemi, M. M.; Zirak, M. Eur. J. Org. Chem. 2006, 5152.

61. Sampath, K. H. M.; Subba-reddy, B. V.; Tirupathi, R. P.; Yadav, J. S. Synthesis, 1999, 4, 586.

62. Gallo, G. G.; Sensi, P. Ann. Chim. (Rome), 1956, 46, 816.

63. Saednya, A. Synthesis, 1983, 9, 748.

64. Nitin, D. A.; Dinesh, S. B.; Krishnacharya, G. A. Tetrahedron Lett., 2007, 48, 3651.

65. Sandmeyer, T. Chem. Ber., 1885, 18, 1495.

66. Sosnovsky, G.; Konieczny, H. T. Z. Naturforsch. B, Anorg. Chem. Org. Chem., 1977, 32, 1179.

67. Beck, G. Justus Liebigs Ann. Chem., 1968, 716, 47.

68. Gronowitz, S.; Sorlin, G.; Gestblom, B.; Hoefman, R. A. Ark. Kemi, 1962, 19, 483.

69. Sampath Kumar, H. M.; Mohanty, P. K.; Suresh Kumar, M.; Yadav, J. S. Synth. C Commun., 1997, 27, 1327.

70. Sharghi, H.; Hosseini Sarvari, M. Tetrahedron, 2002, 58, 10323.

71. Fizet, C.; Streith, J. Tetrahedron Letters, 1974, 15, 3187.

72. Movassagh, B.; Shokria, S. Tetrahedron Letters, 2005, 46, 6923.

73. Miller, M. J.; Loudon, G. M. J. Org. Chem., 1975, 40, 126. 